\title{
Article \\ Evaluation of Crystalline Volume Fraction of Laser-Annealed Polysilicon Thin Films Using Raman Spectroscopy and Spectroscopic Ellipsometry
}

\author{
Jeongsang Pyo, Bohae Lee and Han-Youl Ryu *(D) \\ Department of Physics, Inha University, Incheon 22212, Korea; vywjdtkd789@naver.com (J.P.); \\ bbosea@inha.edu (B.L.) \\ * Correspondence: hanryu@inha.ac.kr
}

Citation: Pyo, J.; Lee, B.; Ryu, H.-Y. Evaluation of Crystalline Volume

Fraction of Laser-Annealed

Polysilicon Thin Films Using Raman Spectroscopy and Spectroscopic Ellipsometry. Micromachines 2021, 12, 999. https://doi.org/10.3390/ mi12080999

Academic Editor: Costas Charitidis

Received: 15 July 2021

Accepted: 20 August 2021

Published: 22 August 2021

Publisher's Note: MDPI stays neutral with regard to jurisdictional claims in published maps and institutional affiliations.

\begin{abstract}
We investigated the crystallinities of poly silicon (poly Si) annealed via green laser annealing (GLA) with a 532-nm pulsed laser and blue laser annealing (BLA) with 450-nm continuous-wave lasers. Three-dimensional heat transfer simulations were performed to obtain the temperature distributions in an amorphous silicon ( $a$-Si) thin film, and GLA and BLA experiments were conducted based on the thermal simulation results. The crystallinity of annealed poly Si samples was analyzed using Raman spectroscopy and spectroscopic ellipsometry. To evaluate the degree of crystallization for the annealed samples quantitatively, the measured spectra of laser-annealed poly Si were fitted to those of crystalline $\mathrm{Si}$ and $a-\mathrm{Si}$, and the crystal volume fraction $\left(f_{c}\right)$ of the annealed poly Si sample was determined. Both the Raman spectroscopy and ellipsometry showed consistent results on $f_{c}$. The $f_{c}$ values were found to reach $>85 \%$ for optimum laser power of GLA and BLA, showing good crystallinity of the laser-annealed poly Si thin films comparable to thermal furnace annealing.
\end{abstract}

Keywords: laser anneal; crystallization; low-temperature poly silicon; Raman spectroscopy; ellipsometry

\section{Introduction}

Laser annealing has been widely adopted as a low-temperature polycrystalline silicon (LTPS) process to crystalize amorphous silicon ( $a$-Si) materials into polycrystalline silicon (poly Si) grains. Poly Si films with high mobility can be achieved by the LTPS process, leading to the realization of low-power thin-film transistor (TFT) panels in liquid crystal or organic light-emitting diode displays [1,2]. In the laser annealing process, the temperature increase is limited only to a low irradiated volume of a sample, which facilitates an extremely localized LTPS process in microtechnology [3-5]. Recently, the use of laser annealing has attracted considerable attention for three-dimensional (3-D) sequential architectures in semiconductors, where the thermal budget for annealing processes is drastically limited [6,7].

In the laser annealing process for a large-area TFT panel, excimer laser annealing (ELA) using ArF (197 nm), KrF (308 nm), or XeCl (308 nm) lasers have been widely employed owing to their high laser power and large absorption coefficient for $a$-Si [8-11]. However, ELA has the issues of high maintenance cost and material non-uniformity. In recent years, various laser annealing technologies have been developed for LTPS processes as alternatives to ELA: green laser annealing (GLA) [12-15], near-ultraviolet laser annealing (NULA) [16-19], and blue laser annealing (BLA) [20-22]. GLA and NULA usually employed $Q$-switched diode-pumped solid-state (DPSS) lasers emitted at 532 and $355 \mathrm{~nm}$, respectively. In BLA, high-power continuous-wave $(\mathrm{CW})$ blue laser diodes emitted around $450 \mathrm{~nm}$ have been used. DPSS and diode lasers have the advantages of high stability and low maintenance as well as installation costs over excimer lasers. Up until now, good crystallization properties have been reported in poly Si films fabricated using GLA, NULA, and BLA. However, the degree of crystallization has not been quantitatively analyzed in 
laser-annealed poly Si samples. Therefore, it was not easy to compare the crystal quality of laser-annealed poly Si to that of poly Si samples fabricated by other annealing processes or poly Si samples annealed with different laser sources.

In this paper, we report a quantitative evaluation of the crystallinities of laser-annealed poly Si thin films using Raman spectroscopy and spectroscopic ellipsometry. A 100-nmthick $a$-Si film deposited on $\mathrm{SiO}_{2} / \mathrm{Si}$ wafer was used for laser annealing of this study. First, the temperature distributions during laser annealing processes were investigated using a thermal simulation with the COMSOL Multiphysics program to determine the conditions of optimum laser power [23]. Based on the simulation results, laser annealing experiments were conducted on the $a$-Si film using GLA and BLA. In addition, thermal furnace annealing was performed to compare the crystallinities of the laser-annealed samples. Although furnace annealing for $a$-Si is known to show good crystallinity, it requires a high temperature and long process time.

Subsequently, the degree of crystallization for the laser-annealed and furnace-annealed samples was analyzed using Raman spectroscopy and spectroscopic ellipsometry. These are nondestructive optical methods that have frequently been employed to analyze the characteristics of annealed poly Si. Spectroscopic ellipsometry can provide information on the optical constants and film thickness of a poly Si layer [24-26]. Raman spectroscopy has the advantages of a simple measurement setup and short measurement time. It has been adopted for the quantitative analysis of crystallinity of poly Si samples fabricated by thermal annealing [27-29].

Quite recently, we reported the comparison of the crystallinity of poly $\mathrm{Si}$ annealed via NULA, BLA, and GLA at one specific laser power level using the spectroscopic ellipsometry [30]. However, few studies have investigated Raman spectroscopy for the quantitative analysis of the crystallization by laser annealing. In this work, for quantitative evaluation of crystallization, the Raman and optical constant spectra of annealed samples were fitted to those of crystalline silicon $(c-\mathrm{Si})$ and $a$-Si, and the crystal volume fraction $\left(f_{c}\right)$ of the annealed poly Si samples was determined for three different laser power levels. It will be revealed that Raman spectroscopy showed consistent results with spectroscopic ellipsometry for all laser power levels, implying that Raman spectroscopy can be conveniently and reliably employed for evaluating the crystal quality of laser-annealed poly Si.

\section{Methods}

\subsection{Thermal Simulation}

Figure 1 schematically shows the $a$-Si sample structure and the laser annealing experiment performed in this study. The sample structure consisted of 100-nm-thick $a$-Si and $\mathrm{SiO}_{2}$ layers prepared via plasma-enhanced chemical vapor deposition (PECVD) on a silicon substrate. The substrate temperature for PECVD was $200^{\circ} \mathrm{C}$. After the deposition of the $a$-Si film, de-hydrogenation was performed at $500{ }^{\circ} \mathrm{C}$ for $30 \mathrm{~min}$ to remove hydrogen content in $a$-Si. During the laser annealing experiment, a laser beam was focused on the sample surface using an objective lens and the beam scanned the sample surface in the $x y$ plane, as shown in Figure 1. The temperature distribution during the laser illumination was investigated using thermal simulations to determine the optimum laser power level in GLA and BLA processes. 


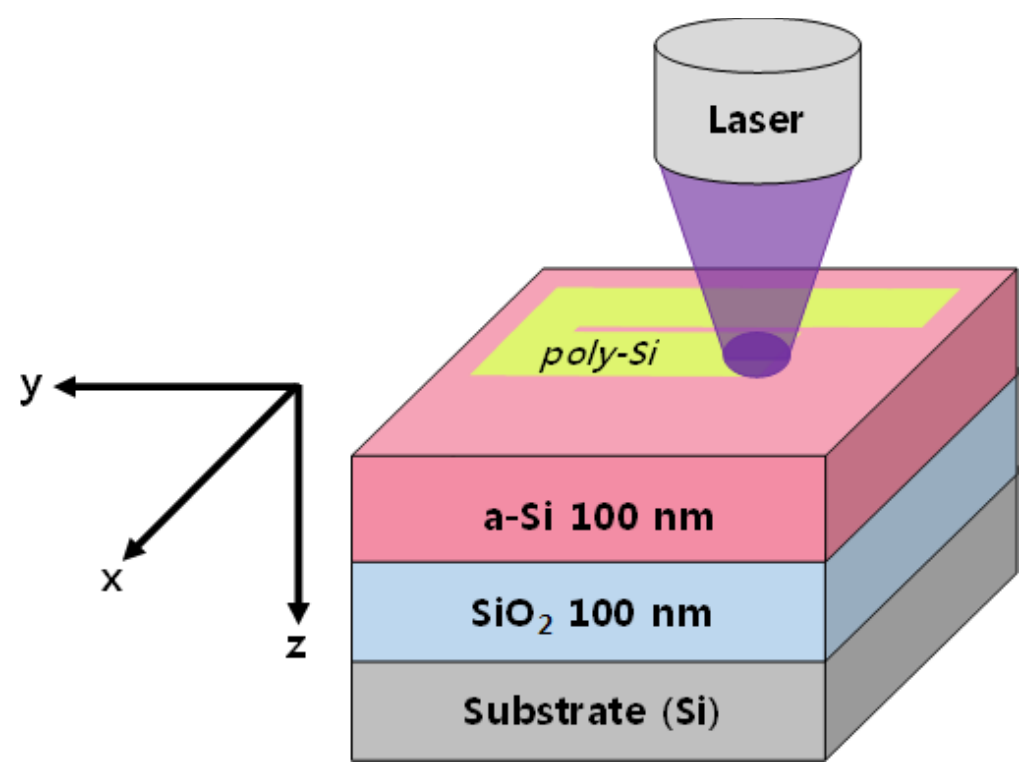

Figure 1. Schematic of the laser annealing experiment for an $a-\mathrm{Si} / \mathrm{SiO}_{2}$ thin film on a single crystalline silicon wafer. The $x-, y-$, and $z$-axes represent the Cartesian coordinate system for heat conduction simulations. The laser beam scans the sample surface in the $y$ direction.

In a non-uniform isotropic medium, three-dimensional (3-D) temperature distribution can be obtained by solving the following heat conduction equation:

$$
\rho C_{p} \frac{\partial T}{\partial t}=\nabla \cdot(\kappa \nabla T)+Q(x, y, z, t)
$$

where $\rho$ is the mass density, $C_{p}$ is the specific heat capacity, $T$ is the temperature, $\kappa$ is the thermal conductivity, and $Q(x, y, z, t)$ is the volumetric heat source [31-33]. Variables $x, y$ and $z$ represent the axes of the coordinate system shown in Figure 1, and $t$ is the time. In this study, the heat conduction equation in Equation (1) was solved numerically using the COMSOL Multiphysics program [23].

When the laser light is absorbed by the sample, the light intensity decreases exponentially with the propagation distance. Therefore, according to the Beer-Lambert law, $Q$ can be written as

$$
Q(x, y, z, t)=-\alpha I(x, y, t)(1-R) e^{-\alpha z},
$$

where $\alpha$ is the absorption coefficient of the material, $R$ is the reflectivity of the sample, and $I(x, y, t)$ is the intensity distribution of the laser. The spatial distribution of $I(x, y, t)$ was assumed to have a Gaussian shape for GLA and a rectangular shape for BLA in the $x y$ plane according to the actual spatial distribution of each laser. The temporal distribution of $I(x, y, t)$ for GLA was also assumed to have a Gaussian shape with the full width half maximum (FWHM) of $30 \mathrm{~ns}$ based on the measured pulse shape of the laser. In addition, the following equations were used for the convective cooling and radiation conditions:

$$
\mathbf{n} \cdot(\kappa \nabla T)=h\left(T_{\mathrm{amb}}-T\right) \text { and } \mathbf{n} \cdot(\kappa \nabla T)=\varepsilon k\left(T_{\mathrm{amb}}^{4}-T^{4}\right),
$$

where $h, T_{\mathrm{amb}}, \varepsilon$, and $k$ are the convection constant, ambient temperature of the processing atmosphere, thermal emission coefficient, and Boltzmann constant, respectively. $\mathbf{n}$ is the normal vector toward exterior of the boundary. $T_{\text {amb }}$ was set at $25^{\circ} \mathrm{C}$ in simulations, and the substrate temperature of samples was also set to be the same as $T_{\mathrm{amb}}$. For the value of $h$, we chose $10 \mathrm{~W} / \mathrm{m}^{2} \mathrm{~K}$, which roughly corresponds to $h$ of air. However, the relative contribution of the convection and radiation to the temperature distribution inside the sample was found to be $<0.5 \%$. The material parameters used for the simulation are listed in Table 1, which were adopted from Refs. [31-34]. 
Table 1. Material parameters used for thermal simulation.

\begin{tabular}{|c|c|c|}
\hline Parameter & Type & Value \\
\hline \multirow{3}{*}{ Density $(\rho)$} & $c-S i$ & $2329 \mathrm{~kg} / \mathrm{m}^{3}$ \\
\hline & $a-S i$ & $2260 \mathrm{~kg} / \mathrm{m}^{3}$ \\
\hline & $\mathrm{SiO}_{2}$ & $2203 \mathrm{~kg} / \mathrm{m}^{3}$ \\
\hline \multirow{3}{*}{ Melting temperature } & $a-\mathrm{Si}$ & $1420 \mathrm{~K}$ \\
\hline & $\mathrm{SiO}_{2}$ & $1986 \mathrm{~K}$ \\
\hline & $c-\mathrm{Si}^{-}$ & $700 \mathrm{~J} /(\mathrm{kg} \cdot \mathrm{K})$ \\
\hline \multirow{4}{*}{ Heat capacity $\left(C_{p}\right)$} & $a-\mathrm{Si}$ & $992 \mathrm{~J} /(\mathrm{kg} \cdot \mathrm{K})$ \\
\hline & $\mathrm{SiO}_{2}$ & $703 \mathrm{~J} /(\mathrm{kg} \cdot \mathrm{K})$ \\
\hline & Melted Si & $965 \mathrm{~J} /(\mathrm{kg} \cdot \mathrm{K})$ \\
\hline & $c-\mathrm{Si}$ & $130 \mathrm{~W} / \mathrm{m} \cdot \mathrm{K}$ \\
\hline \multirow{3}{*}{$\begin{array}{l}\text { Thermal conductivity } \\
\qquad(\kappa)\end{array}$} & $a-S i$ & $1.5 \mathrm{~W} / \mathrm{m} \cdot \mathrm{K}$ \\
\hline & $\mathrm{SiO}_{2}$ & $1.38 \mathrm{~W} / \mathrm{m} \cdot \mathrm{K}$ \\
\hline & Melted Si & $2 \mathrm{~W} / \mathrm{m} \cdot \mathrm{K}$ \\
\hline \multirow{2}{*}{ Reflectivity $(R)$} & 450-nm laser & 0.483 (a-Si), 0.7(Melted Si) \\
\hline & 532-nm laser & 0.403 (a-Si), 0.71 (Melted Si) \\
\hline Surface emissivity & $a-S i$ & 0.5 \\
\hline coefficient $(\varepsilon)$ & Melted Si & 0.7 \\
\hline Latent heat & $a-\mathrm{Si}$ & $1789.42 \mathrm{~J} / \mathrm{g}$ \\
\hline Convection constant $(h)$ & air & $10 \mathrm{~W} / \mathrm{m}^{2} \mathrm{~K}$ \\
\hline Absorption & 450-nm laser & $0.0436 \mathrm{~nm}^{-1}$ \\
\hline coefficient $(\alpha)$ & 532-nm laser & $0.0207 \mathrm{~nm}^{-1}$ \\
\hline
\end{tabular}

In Reference [30], thermal simulation for laser annealing was also performed using COMSOL Multiphysics and the temperature distribution for one specific power condition was presented. Compared to the results in Reference [30], in this work, the thermal simulation was performed more systematically for BLA and GLA, including temporal and spatial distributions, and the temperature distributions for different power levels were investigated to find the optimum power condition.

\subsection{Annealing Experiment}

The laser annealing equipment was constructed to perform GLA and BLA. The $a$-Si sample for annealing was placed on a computer-controlled $x-y$ stage. The sample dimension for the annealing experiment was about $1 \times 1 \mathrm{~cm}^{2}$. The temperature of the sample stage was the same as the ambient temperature of $25^{\circ} \mathrm{C}$. In the laser annealing experiment, a laser beam was focused on the sample surface using an objective lens and it scanned the sample surface in the $x y$ plane by moving the sample stage, as shown in Figure 1. The scanning speed was chosen to be $4 \mathrm{~cm} / \mathrm{s}$, which was expected to generate a reasonably uniform temperature distribution in the scanning direction. For the 532-nm laser in GLA, the second harmonic of a $Q$-switched DPSS Nd:YAG laser was employed. The pulse repetition rate and pulse width were $30 \mathrm{kHz}$ and $30 \mathrm{~ns}$, respectively. When the laser was illuminated on the sample surface, the beam profile was approximately measured to be a Gaussian function with the beam waist of $\sim 6 \mu \mathrm{m}$. The CW laser for BLA consisted of two GaN-based blue laser diodes emitted at $450 \mathrm{~nm}$. The laser beam of BLA has a rectangular shape with a spot size of $6 \mu \mathrm{m} \times 2 \mu \mathrm{m}$. The laser power was monitored using a laser power meter during the annealing process. The laser annealing experiments were performed under nitrogen environment. The sample for laser annealing was placed inside a small plastic container, where the nitrogen gas was filled. The pressure of nitrogen was maintained at 1.5 times the atmospheric pressure.

For GLA, the average power of lasers was measured from the power meter, and the peak power was determined using the pulse width and pulse repetition rate. The laser annealing experiments were mainly performed at the peak power of $2.7 \mathrm{~W}$ for GLA and a CW power of $530 \mathrm{~mW}$ for BLA. At these laser powers, the temperature at the sample surface reached $\sim 2900 \mathrm{~K}$ based on the thermal simulation result, which will be shown later. Assuming the Gaussian shape in the spatial and temporal distribution of pulsed lasers, the 
peak power of $2.7 \mathrm{~W}$ for GLA corresponds to the pulse energy of $86 \mathrm{~nJ}$ and energy density of $153 \mathrm{~mJ} / \mathrm{cm}^{2}$. In addition to GLA and BLA, thermal furnace annealing was performed to compare the crystallinities of the laser-annealed poly Si samples. In the furnace annealing experiment, the $a$-Si sample was annealed for $30 \mathrm{~min}$ at $800{ }^{\circ} \mathrm{C}$.

Figure 2 shows atomic force microscopy (AFM) images of an $a$-Si sample before annealing, poly Si samples annealed via furnace annealing, BLA, and GLA. The AFM images were measured on a sample area of $5 \times 5 \mu \mathrm{m}^{2}$. The root-mean-square (RMS) roughness of the samples was determined from the AFM images in Figure 2. The RMS roughness was $0.3,1.2,4.5$, and $5.7 \mathrm{~nm}$ for $a$-Si before annealing, furnace annealing, BLA, and GLA, respectively.

(a)

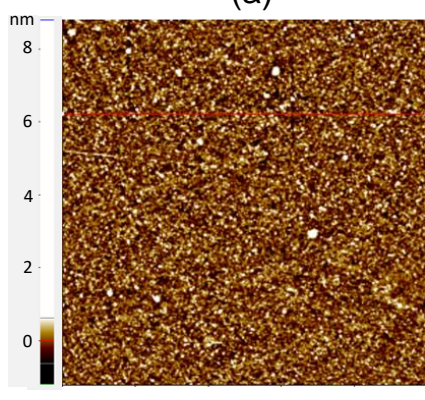

(b)

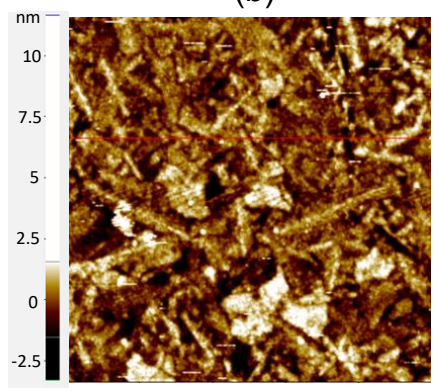

(c)

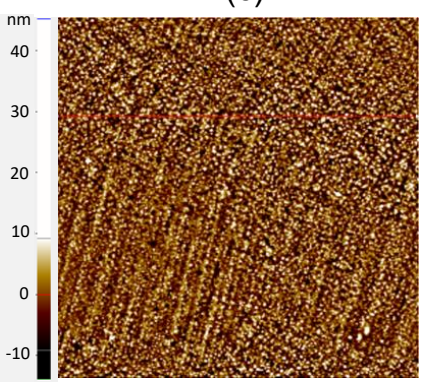

(d)

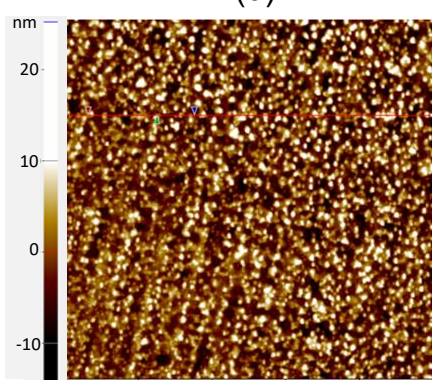

Figure 2. AFM images of a sample area of $5 \times 5 \mu \mathrm{m}^{2}$ for (a) an $a$-Si sample before annealing, (b) a furnace-annealed poly $\mathrm{Si}$ sample, (c) a poly Si sample annealed via BLA, and (d) a poly Si sample annealed via GLA.

\section{Results and Discussion}

\subsection{Thermal Simulation Results}

Using Equations (1)-(3) and the material parameters in Table 1, 3-D thermal simulations were performed to obtain temperature distributions in the $x y$ plane and in the $z$ direction as the laser power varied. The simulation area was $20 \times 20 \mu \mathrm{m}^{2}$, which is sufficiently larger than actual laser beam spot area. Figure 3a shows the temporal variation of the temperature at a certain point on the sample surface as the laser beam scans the sample. The temperature is plotted as a function of time for GLA with the peak power of $2.7 \mathrm{~W}$ and BLA with the $\mathrm{CW}$ power of $530 \mathrm{~mW}$. These laser powers correspond to the maximum surface temperature of $\sim 2900 \mathrm{~K}$. For GLA, the temperature increased rapidly and reached a peak value, and then it decreased to the ambient temperature. This temporal response of the temperature resulted from the pulsed operation of the 532-nm laser. For BLA, on the contrary, the temperature increased slowly with time and reached a constant value because of the $\mathrm{CW}$ operation of the 450 -nm laser.

Figure $3 \mathrm{~b}$ shows the surface temperature of the $a$-Si layer as a function of the sample length in the scanning direction (the $y$ direction in Figure 1) for three different scanning speeds of GLA. The temperature in Figure $3 \mathrm{~b}$ corresponds to the maximum temperature in the temporal variation of temperature in Figure 3a. Here, the peak power of the $532-\mathrm{nm}$ green laser was set to $2.7 \mathrm{~W}$. When a pulsed laser scans the sample surface, the temperature in the scanning direction becomes inhomogeneous, depending on the pulse duration and repetition time. The temperature difference increased with the increase in the scanning speed, as shown in Figure $3 \mathrm{~b}$. When the scanning speed was $16 \mathrm{~cm} / \mathrm{s}$, the highest and lowest temperatures on the sample surface were 2870 and $1980 \mathrm{~K}$, respectively. The difference in the surface temperatures was $\sim 900 \mathrm{~K}$, which could result in a significant non-uniformity in the crystallinity. At a scanning speed of $10 \mathrm{~cm} / \mathrm{s}$, the temperature difference was $\sim 400 \mathrm{~K}$, which was less than half of that at a scanning speed of $16 \mathrm{~cm} / \mathrm{s}$. When the scanning speed was further decreased to $4 \mathrm{~cm} / \mathrm{s}$, the surface temperature varied from 2780 to $2900 \mathrm{~K}$. That is, the temperature difference was reduced to $120 \mathrm{~K}$, which is expected to yield a considerably improved uniformity in the crystallinity. From the result of Figure $3 b$, it is expected that the crystallinity of the laser-annealed poly Si film is improved 
as the scanning speed decreases, at the expense of a long laser processing time. In this study, we selected $4 \mathrm{~cm} / \mathrm{s}$ as the scanning speed of GLA to obtain uniform crystallinity. For BLA, the temperature in the scanning direction was found to be almost uniform because of the CW operation of blue lasers.

(a)

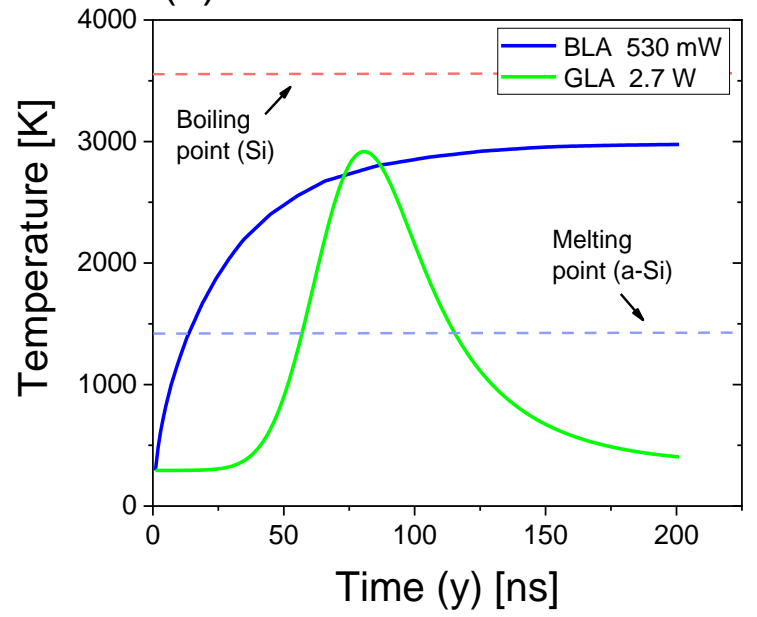

(b)

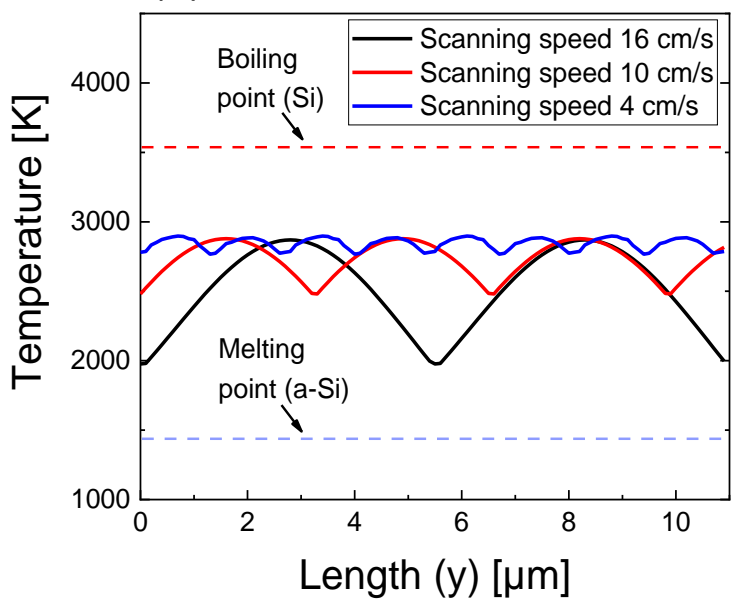

Figure 3. (a) Temperature on the sample surface as a function of time for BLA with the CW power of $530 \mathrm{~mW}$ and GLA with the peak power of $2.7 \mathrm{~W}$. (b) Temperature profiles on the sample surface in the laser scanning direction ( $y$ direction in Figure 1) for GLA with the peak power of $2.7 \mathrm{~W}$ when the scanning speed is 4,10 , and $16 \mathrm{~cm} / \mathrm{s}$.

Figure 4 shows the simulated temperature distributions as a function of the sample depth (the $z$ direction in Figure 1) for three different power levels of GLA and BLA. The temperature decreased steadily with increasing depth. The temperature at the interface between $a$-Si and $\mathrm{SiO}_{2}$ should be higher than the melting point of $a$-Si $(1420 \mathrm{~K})$ for crystallizing the $a$-Si layer. However, the temperature on the surface of the $a$-Si layer should be lower than the boiling point of silicon $(3538 \mathrm{~K})$ to avoid the vaporization of the annealed poly $\mathrm{Si}$. In addition, the temperature of the underlying $\mathrm{SiO}_{2}$ layer should be lower than the melting point of $\mathrm{SiO}_{2}(1986 \mathrm{~K})$ to protect it from thermal damage. Therefore, the optimum power condition should be chosen such that the temperature on the sample surface is lower than the boiling point of $\mathrm{Si}$, and the temperature at the $a-\mathrm{Si} / \mathrm{SiO}_{2}$ interface is higher than the melting point of $a$-Si and lower than the melting point of $\mathrm{SiO}_{2}$.
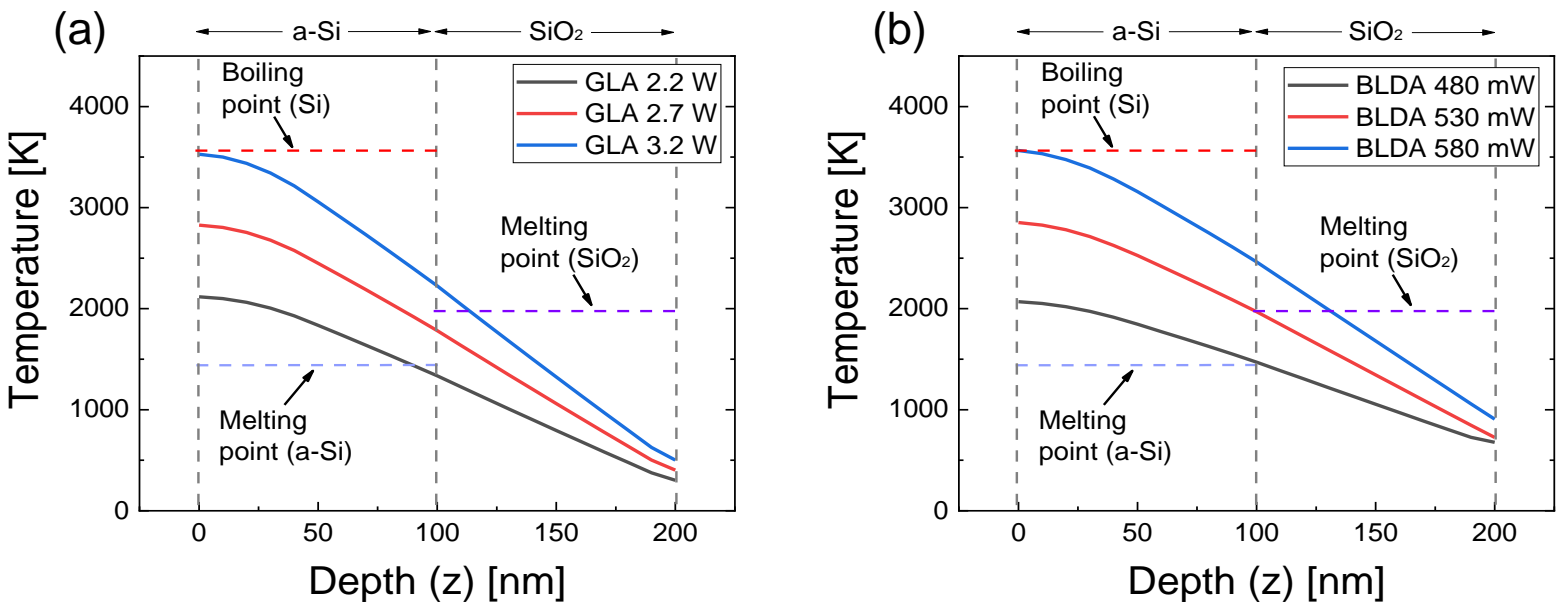

Figure 4. Simulated temperature profile in the direction of the sample depth (the $z$ direction in Figure 1) for (a) GLA with the laser peak powers of 2.2, 2.7, and 3.2 W and (b) BLDA with the CW laser powers of 480, 530, and $580 \mathrm{~mW}$. Here, the maximum temperature in the temporal profile in Figure $3 a$ is plotted at each $z$ point. 
In Figure 4, the surface temperature corresponded to the boiling point of Si for the laser power of $580 \mathrm{~mW}$ for BLA and $3.2 \mathrm{~W}$ for GLA. For the laser powers of $530 \mathrm{~mW}$ for BLA and 2.7 W for GLA, the surface temperature was approximately $2900 \mathrm{~K}$, which was significantly higher than the melting point of $a$-Si and sufficiently lower than the boiling point of $\mathrm{Si}$. For these laser powers, the temperature at the $a-\mathrm{Si} / \mathrm{SiO}_{2}$ interface was well above the melting point of $a$-Si and below the melting point of $\mathrm{SiO}_{2}$ for both BLA and GLA. Therefore, we believe these laser powers could be close to the optimum condition for achieving good crystallinity. When the laser power decreased to $480 \mathrm{~mW}$ for BLA and $2.2 \mathrm{~W}$ for GLA, the surface temperature was $\sim 2000 \mathrm{~K}$, which was still higher than the melting point of $a$-Si. However, for these laser powers, the temperature at the $a-\mathrm{Si} / \mathrm{SiO}_{2}$ interface was just similar to the melting point of $a$-Si, which could result in an incomplete crystallization of $a$-Si.

\subsection{Raman Spectoscopy}

After the annealing processes, the crystallinity of the annealed poly $\mathrm{Si}$ was investigated using Raman spectroscopy and spectroscopic ellipsometry. Raman spectroscopy has been widely employed for investigating the crystal quality of poly Si [28-30]. Raman spectrum for each sample was measured using a micro Raman system (UniDRON-3200, UniNano Tech Co., Yongin-si, Korea). In the Raman spectroscopy system, a CW 532-nm laser with a power of $25 \mathrm{~mW}$ and a spot size of $\sim 3 \mu \mathrm{m}$ was adopted. The resolution of Raman shift was $0.5 \mathrm{~cm}^{-1}$. Figure 5 a shows the Raman spectra of annealed poly $S i$ samples along with those of $a$-Si and crystalline silicon (c-Si) samples. The Raman spectrum of $a$-Si shows a broad spectral distribution centered at approximately $480 \mathrm{~cm}^{-1}$ associated with transverse optic (TO) phonons. In contrast, $c$-Si shows a sharp peak in the TO phonon mode at approximately $520 \mathrm{~cm}^{-1}$. The crystallinity of the annealed poly Si film can be analyzed using the peak shift and FWHM of $a$-Si and c-Si. As the crystallinity improves, the peak in the TO phonon mode of poly Si shifts towards that of $c$-Si. The peak Raman shift for the furnace annealing, BLA, and GLA was $519,517.5$, and $516.5 \mathrm{~cm}^{-1}$, respectively. The FWHM for the furnace annealing, BLA, and GLA was 7.5, 9.0, and $9.5 \mathrm{~cm}^{-1}$, respectively. Therefore, from the Raman spectra in Figure $5 \mathrm{a}$, it is expected that the furnace-annealed poly Si sample will show better crystallinity than the laser-annealed poly Si samples, and BLA will show slightly better crystallinity than GLA.

Recently, the crystal volume fraction $\left(f_{c}\right)$ of poly $\mathrm{Si}$ was evaluated using the relative values of FWHM and intensity using TO phonon mode of the materials in the Raman spectrum [35]. $f_{\mathcal{c}}$ of poly $\mathrm{Si}$ can be quantitatively evaluated using the following formula:

$$
f_{c}=\frac{I_{c}}{I_{c}+I_{a}}
$$

where $f_{c}$ represents the crystalline volume fraction. $I_{c}$ and $I_{a}$ indicate the integrated Raman scattering intensity of crystalline and amorphous sections, respectively. $I_{c}$ and $I_{a}$ can be obtained by the deconvolution of each Raman spectrum into Gaussian components corresponding to the crystalline and amorphous phases, respectively.

Figure $5 b-d$ shows the fit of Raman spectra of poly Si films formed via furnace annealing, GLA, and BLA, respectively. In this case, the peak power of GLA was $2.7 \mathrm{~W}$ and the CW power of BLA was $530 \mathrm{~mW}$. Each Raman spectrum was decomposed into three Gaussian functions corresponding to one amorphous phase and two crystalline parts of a weak crystalline phase and a full crystalline phase [35]. According to Ref. [35], the two crystalline phases can be attributed to nanocrystalline silicon and crystalline silicon phases, respectively. The peak positions of three Gaussian functions centered around 498, 516, and $518 \mathrm{~cm}^{-1}$. In Figure 5, the Gaussian functions of the peaks 1 and 2 correspond to $I_{c}$ and that of the peak 3 corresponds to $I_{a}$ in Equation (4), respectively. The measured Raman spectrum (black solid line) and the cumulative fit curve (red dotted line) of the three Gaussian functions show good consistency for all cases. As a-Si becomes crystallized to poly $\mathrm{Si}$, the TO phonon peak of the crystalline phase is shifted slowly toward the peak 
of $c$-Si and the FWHM is decreased, resulting in an increase in $f_{c}$. From the fitting results, $f_{c}$ was calculated using Equation (4). The $f_{c}$ value of poly Si formed via furnace annealing was $96.2 \%$, which was the highest value among all annealed samples. The $f_{c}$ values for BLA and GLA were $90.6 \%$ and $88.2 \%$, respectively, implying that the poly Si formed via BLA showed slightly higher crystal quality than that formed via GLA. This is consistent with the observation that BLA showed the longer Raman peak shift and the narrower FWHM than GLA as shown in Figure 5a. The crystal volume fraction values relative to furnace annealing were $94.1 \%$ and $91.7 \%$ for BLA and GLA, respectively, indicating that the laser-annealed poly $\mathrm{Si}$ showed crystallinity comparable to thermal furnace-annealed poly Si.
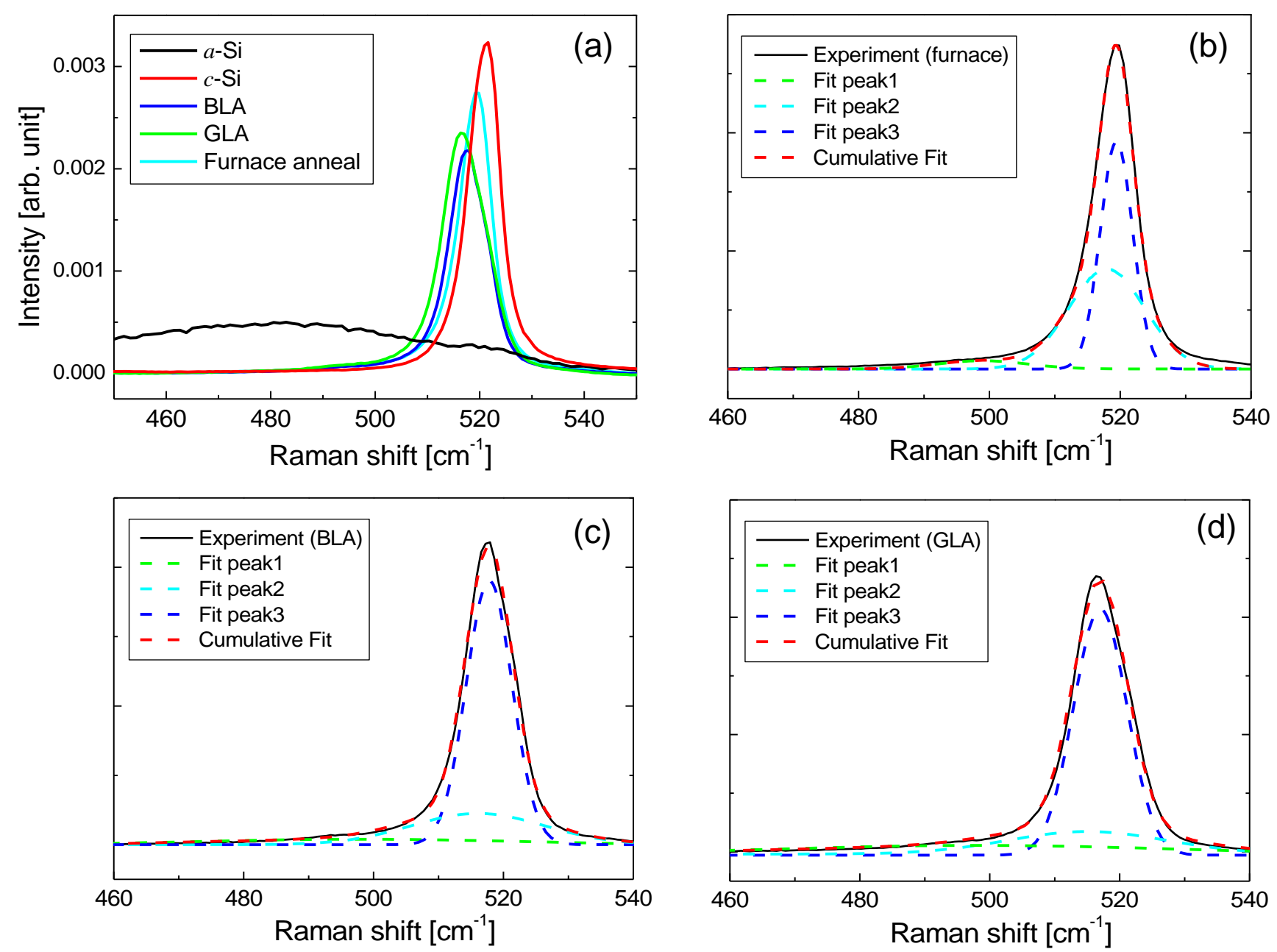

Figure 5. (a) Raman spectra of $a-\mathrm{Si}, c-\mathrm{Si}$, and poly silicon samples with BLA, GLA, and thermal furnace annealing. (b-d) Fit of Raman spectra of poly Si films formed via furnace annealing, GLA, and BLA, respectively. The experimental spectrum is represented by the black solid lines. The green, cyan, and blue dotted lines represent the fitted three Gaussian curves. The red lines represent the cumulative fit curves.

To investigate the effect of laser power levels on crystallinity, laser annealing experiments were performed at other laser power levels that corresponded to the laser powers of thermal simulations in Figure 4 . Then, the $f_{c}$ values were determined for each power level using the Raman spectrum analysis. In Figure 6 , the $f_{c}$ values obtained by the Raman spectrum analysis are plotted. The three power levels $P_{1}, P_{2}, P_{3}$ respectively correspond to 2.2, 2.7, $3.2 \mathrm{~W}$ for GLA and 480, 530, $580 \mathrm{~mW}$ for BLA. As the laser power increased from $P_{1}$ to $P_{3}, f_{c}$ increased from $80.8 \%$ to $90.2 \%$ for GLA and from $82.9 \%$ to $93.7 \%$ for BLA. For all laser power levels, BLA showed slightly higher $f_{c}$ values than GLA. The result in Figure 6 shows that the crystallinity of laser-annealed poly Si was improved as the laser power 
increased. At $P_{3}$, BLA and GLA resulted in $f_{c}$ of $>90 \%$, which is close to $f_{c}$ of the thermal furnace annealing. However, it was found that surface roughness increased significantly at $P_{3}$, implying partial boiling of Si might occur at a power level this high.

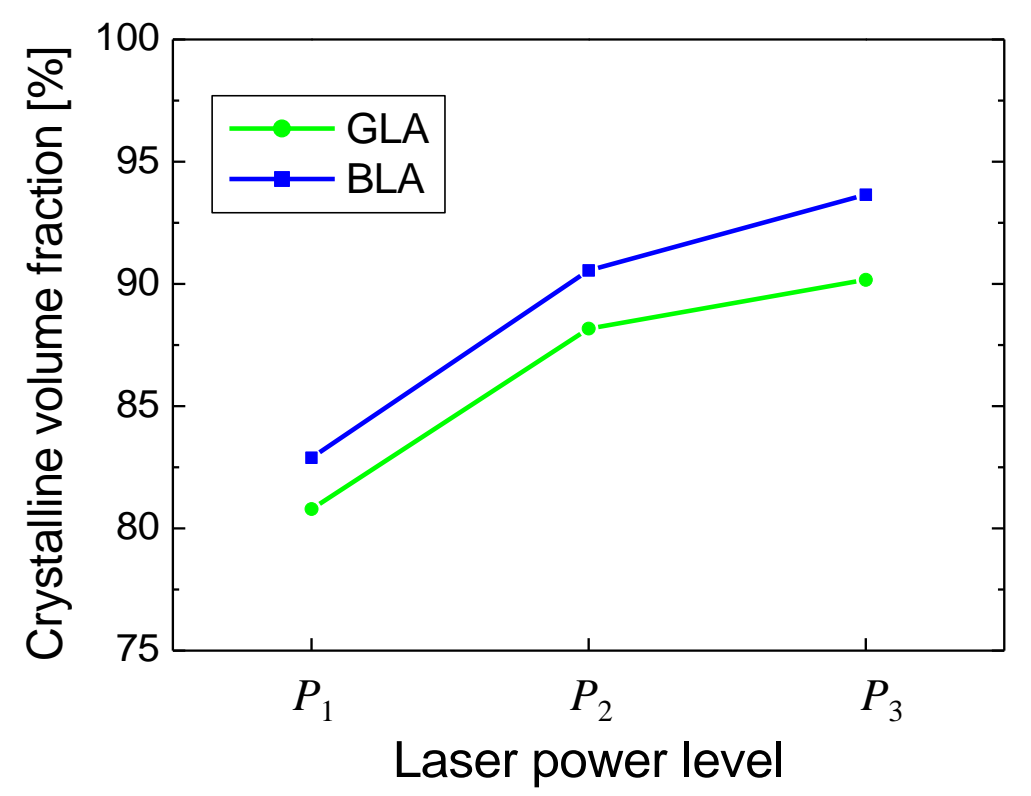

Figure 6. Crystal volume fraction $\left(f_{c}\right)$ for BLA and GLA at the three laser power levels shown in Figure 4 . The laser power levels $P_{1}, P_{2}, P_{3}$ respectively correspond to 480, 530, $580 \mathrm{~mW}$ for BLDA and $2.2,2.7,3.2 \mathrm{~W}$ for GLA.

\subsection{Spectroscopic Ellipsometry}

The crystallinities of the annealed poly Si samples were also analyzed quantitatively using the optical constant spectra obtained by spectroscopic ellipsometry measurements. For the measurement, we employed a spectroscopic ellipsometer (V-VASE, JAWoollam Co., Lincoln, NE, USA) at a fixed angle of $70^{\circ}$ in a wavelength range of 300 to $1600 \mathrm{~nm}$. In ellipsometry, the amplitude and phase difference between two polarization states, which is often denoted as $\Psi$ and $\Delta$, are measured for the light reflecting from the surface of a thin film. The refractive index $(n)$ and extinction coefficient $(k)$ spectra can be obtained by the fit of experimental $\Psi$ and $\Delta$ spectra. The effective medium approximation (EMA) method was employed for the fitting [36,37].

For the EMA process, a poly Si film was assumed to be composed of $a$-Si and $c$-Si. The crystalline volume fraction of the annealed poly $\mathrm{Si}$ can be obtained using the EMA method. Among the EMA methods, the Bruggeman-type method has been widely employed to estimate the volume fraction of composite materials for dielectric multilayers, and can be expressed as $[36,37]$

$$
f_{a} \frac{\varepsilon_{a}-\varepsilon_{\text {poly }}}{\varepsilon_{a}+2 \varepsilon_{\text {poly }}}+f_{c} \frac{\varepsilon_{c}-\varepsilon_{\text {poly }}}{\varepsilon_{c}+2 \varepsilon_{\text {poly }}}=0 \text { and } f_{a}+f_{c}=1,
$$

where $\varepsilon_{a}$ and $\varepsilon_{c}$ are the dielectric functions of $a$-Si and $c$-Si, respectively. $\varepsilon_{\text {poly }}$ is an effective dielectric function of the composite material (poly $\mathrm{Si}$ ). The dielectric function can be correlated with the complex refractive index, which is composed of the refractive index and extinction coefficient. $f_{a}$ and $f_{c}$ are the volume fractions of $a$-Si and $c-\mathrm{Si}$, respectively.

For fitting the experimental $\Psi$ and $\Delta$ data, $f_{c}$ and the film thickness $(d)$ were used as fitting parameters. The fitting results showed that the mean squared error (MSE) was 1 2. This MSE value is much smaller than previous results for similar thin film structures [35,38], indicating excellent data fitting with reliable results on $f_{c}$ and $d$. From the results of the fitting, the $f_{c}$ values of the annealed poly Si were determined to be $88.6 \%$, and $85.6 \%$ for BLA and GLA, respectively. Using the obtained $f_{c}$ value, the $n, k$ spectra of annealed poly 
Si can be obtained from Equation (5). Figure 7 shows $n$ and $k$ spectra of the laser-annealed poly $\mathrm{Si}$ samples, $c-\mathrm{Si}$, and $a$-Si when the peak power of GLA was $2.7 \mathrm{~W}$ and the $\mathrm{CW}$ power of BLA was $530 \mathrm{~mW}$. As the crystallinity improves, the spectral shape of poly Si approaches that of $c$-Si. It can be observed that the $n$ and $k$ spectra of the furnace-annealed poly Si are very close to those of $c$-Si, indicating the good crystal quality of the furnace-annealed sample. The laser-annealed poly $\mathrm{Si}$ samples also exhibit spectral shapes similar to those in the case of $c$-Si.
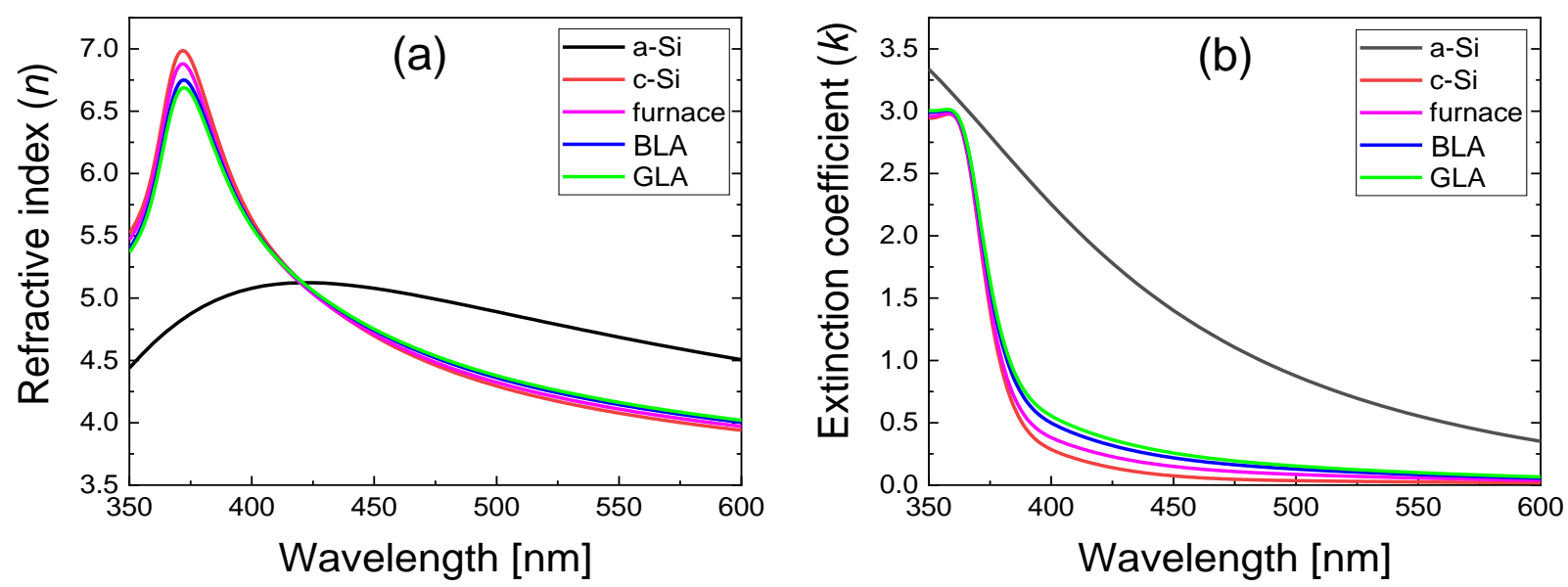

Figure 7. Optical constant spectra of poly Si samples annealed via furnace annealing, BLA, and GLA. The spectra of $a$-Si and $c$-Si are also shown for reference. (a) Refractive index $(n)$ spectra and (b) extinction coefficient $(k)$ spectra.

In Figure 8, the $f_{\mathcal{c}}$ values obtained by Raman spectroscopy and spectroscopic ellipsometry are compared for poly Si samples annealed via furnace annealing, GLA, and BLA when the laser power was $2.7 \mathrm{~W}$ for GLA and $530 \mathrm{~mW}$ for BLA. The results of the two methods show good correlation with a difference of only $2-3 \%$ in the $f_{c}$ value for all poly $\mathrm{Si}$ samples. The $f_{c}$ values obtained by spectroscopic ellipsometry were slightly lower than those obtained by Raman spectroscopy. The ellipsometry measurement also showed that the poly Si formed via BLA had slightly better crystal quality than that formed via GLA similarly to the case of Raman spectroscopy. The results of Figure 8 indicate that the Raman spectra as well as the $n$ and $k$ spectra obtained using ellipsometry can be successfully applied for the quantitative evaluation of the crystallinity of poly $\mathrm{Si}$. The average $f_{c}$ values were $95.4 \%, 89.6 \%$, and $86.9 \%$ for furnace annealing, BLA, and GLA, respectively.

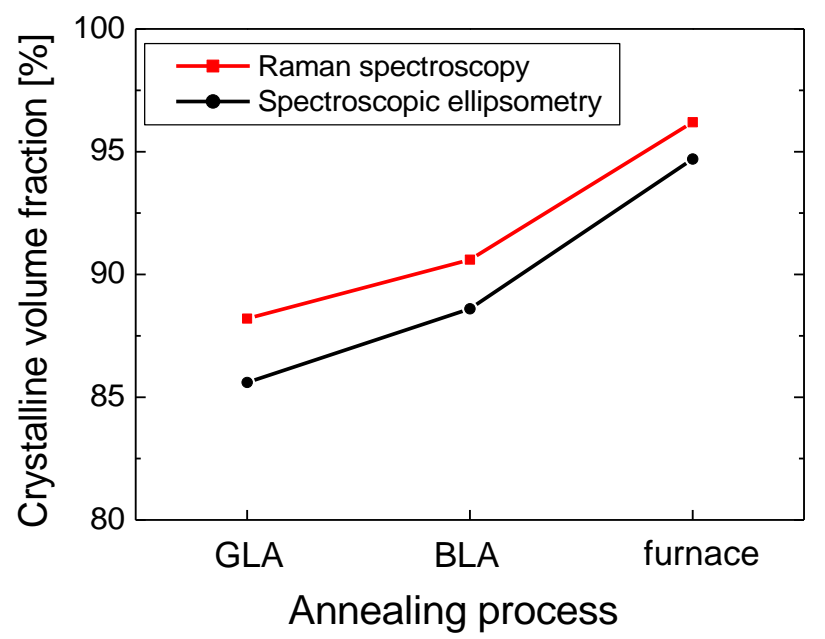

Figure 8. Crystalline volume fraction $\left(f_{c}\right)$ for GLA, BLA, and furnace annealing analyzed using Raman spectroscopy and spectroscopic ellipsometry. 
The analysis results of this study showed that BLA could be better laser annealing process than GLA for the annealing of a 100-nm-thick a-Si layer, which is attributed to the CW laser operation in BLA. Owing to the CW operation, good spatial uniformity is expected. However, thermal damage in the underlayers, which might occur during the $\mathrm{CW}$ laser annealing, should be considered. As shown in the simulation result in Figure 4b, high temperatures of $\sim 730 \mathrm{~K}$ could be maintained even below the $\mathrm{SiO}_{2}$ underlayer. Although GLA showed slightly lower $f_{c}$ than BLA, it also resulted in a good crystallinity with $f_{c}>85 \%$. At the interface between the $\mathrm{SiO}_{2}$ underlayer and the $\mathrm{Si}$ substrate, the temperature of GLA was $400 \mathrm{~K}$, indicating thermal damage to the substrate material could be avoided. In addition, despite the pulsed operation, a reasonably good spatial uniformity is also expected from the simulation results in Figure 3 if the scanning speed is sufficiently slow. The quantitative analysis results presented in this paper imply that the optimum laser-annealing process is expected to realize poly $\mathrm{Si}$ with high crystallinity suitable for LTPS process.

\section{Conclusions}

In summary, we quantitatively evaluated the degree of crystallization of poly $\mathrm{Si}$ thin films annealed via GLA with a 532-nm nanosecond laser and BLA with 450-nm CW laser by determining the crystal volume fraction of annealed poly Si using Raman spectroscopy and spectroscopic ellipsometry. 3-D heat transfer simulations were performed to obtain the temperature distributions during laser annealing processes, and GLA and BLA experiments were conducted based on the thermal simulation results. The $f_{\mathcal{c}}$ values of annealed poly $\mathrm{Si}$ were determined by fitting of the Raman spectrum and the optical constant spectra obtained by ellipsometry. Both the Raman spectroscopy and ellipsometry showed consistent result on the $f_{c}$ values. It was found that the evaluated $f_{c}$ values of laser-annealed poly Si could be as high as $90 \%$, which was comparable to the $f_{c}$ of thermal furnace-annealed poly $\mathrm{Si}$, implying good crystallinity of the laser-annealed poly Si thin films. Because of its simplicity, Raman spectroscopy is expected to be advantageously employed for evaluating the crystal quality of laser-annealed poly Si.

Author Contributions: Conceptualization, J.P. and H.-Y.R.; methodology, J.P. and H.-Y.R.; software, J.P. and B.L.; validation, J.P. and B.L.; formal analysis, J.P.; investigation, J.P. and H.-Y.R.; data curation, H.-Y.R.; writing — original draft preparation, J.P.; writing—review and editing, H.-Y.R.; supervision, H.-Y.R.; project administration, H.-Y.R.; funding acquisition, H.-Y.R. All authors have read and agreed to the published version of the manuscript.

Funding: This research was funded by Nano Material Technology Development Program through the National Research Foundation of Korea (NRF) funded by the Ministry of Science and ICT (2019R1A2C1010160), the Technology Innovation Program (20015909) funded by the Ministry of Trade, Industry \& Energy (MOTIE, Korea), and Korea Institute for Advancement of Technology (KIAT) grant funded by the Korea Government (MOTIE) (P0008458, The Competency Development Program for Industry Specialist).

Data Availability Statement: The data supporting the findings of this paper is available from the corresponding authors upon reasonable request.

Acknowledgments: The authors are grateful to Rino Choi at the Inha University for providing the samples used for the experiments.

Conflicts of Interest: The authors declare no conflict of interest. The funders had no role in the design of the study; in the collection, analyses, or interpretation of data; in the writing of the manuscript, or in the decision to publish the results.

\section{References}

1. Stewart, M.; Howell, R.S.; Pires, L.; Hatalis, M.K. Polysilicon TFT technology for active matrix OLED displays. IEEE Trans. Electron Devices 2001, 48, 845. [CrossRef]

2. Phillips, K.C.; Gandhi, H.H.; Mazur, E.; Sundaram, S.K. Ultrafast laser processing of materials: A review. Adv. Opt. Photonics 2015, 7, 684. [CrossRef] 
3. Theodorakos, I.; Zergioti, I.; Vamvakas, V.; Tsoukalas, D.; Raptis, Y.S. Picosecond and nanosecond laser annealing and simulation of amorphous silicon thin films for solar cell applications. J. Appl. Phys. 2014, 115, 043108. [CrossRef]

4. Franta, B.; Pastor, D.; Gandhi, H.H.; Rekemeyer, P.H.; Gradečak, S.; Aziz, M.J.; Mazur, E. Simultaneous high crystallinity and sub-bandgap optical absorptance in hyperdoped black silicon using nanosecond laser annealing. J. Appl. Phys. 2015, 118, 225303. [CrossRef]

5. Fortunato, G.; Mariucci, L.; Carluccio, R.; Pecora, A.; Foglietti, V. Excimer laser crystallization techniques for polysilicon TFTs. Appl. Surf. Sci. 2000, 154, 95-104. [CrossRef]

6. Huet, K.; Mazzamuto, F.; Tabata, T.; Toqué-Tresonne, I.; Mori, Y. Doping of semiconductor devices by Laser Thermal Annealing. Mater. Sci. Semicon. Proc. 2017, 62, 92-102. [CrossRef]

7. Huet, K.; Aubin, J.; Raynal, E.P.; Curvers, B.; Verstraete, A.; Lespinasse, B.; Mazzamuto, F.; Sciuto, A.; Lombardo, F.S.; La Magna, A.; et al. Pulsed laser annealing for advanced technology nodes: Modeling and calibration. Appl. Surf. Sci. 2020, 505, 144470. [CrossRef]

8. Jhon, Y.M.; Kim, D.H.; Chu, H.; Choi, S.S. Crystallization of amorphous silicon by excimer laser annealing with a line shape beam having a Gaussian profile. Jpn. J. Appl. Phys. 1994, 33, L1438-L1441. [CrossRef]

9. Fujii, M.; Ishikawa, Y.; Ishihara, R.; van der Cingel, J.; Mofrad, M.R.T.; Horita, M.; Uraoka, Y. Low temperature high-mobility InZnO thin-film transistors fabricated by excimer laser annealing. Appl. Phys. Lett. 2013, 102, 122107. [CrossRef]

10. Jang, K.; Kim, Y.; Park, J.; Yi, J. Electrical and structural characteristics of excimer laser-crystallized polycrystalline $\mathrm{Si}_{1-\mathrm{x}} \mathrm{Ge}_{\mathrm{x}}$ thin-film transistors. Materials 2014, 12, 1739. [CrossRef]

11. Choi, D.H.; Kim, H.S.; Oh, S.Y.; Lee, C.H. Drastic improvement of as-sputtered silicon nitride thin film quality at room temperature by ArF excimer-laser annealing method. Curr. Appl. Phys. 2016, 6, 876-885. [CrossRef]

12. Sugawara, Y.; Uraoka, Y.; Yano, H.; Hatayama, T.; Fuyuki, T.; Mimura, A. Crystallization of double-layered silicon thin films by solid green laser annealing for high-performance thin-film transistors. IEEE Electron Dev. Lett. 2007, 28, 395-397. [CrossRef]

13. Kawamura, Y.; Yamasaki, K.; Yamashita, T.; Sugawara, Y.; Uraoka, Y. Crystallization by green-laser annealing for threedimensional device application. J. Korean Phys. Soc. 2010, 56, 1456-1460.

14. Beyer, W.; Andrä, G.; Bergmann, J.; Breuer, U.; Finger, F.; Gawlik, A.; Haas, S.; Lambertz, A.; Maier, C.F.; Nickel, H.N.; et al. Temperature and hydrogen diffusion length in hydrogenated amorphous silicon films on glass while scanning with a continuous wave laser at $532 \mathrm{~nm}$ wavelength. J. Appl. Phys. 2018, 124, 153103. [CrossRef]

15. Sasaki, N.; Arif, M.; Uraoka, Y. Transition mechanism of the thin Si-films obtained by the CW laser lateral crystallization from the grain-boundary free highly $\{100\}$ oriented crystal to the twinned $\{211\}$ crystal depending on the laser power. Jpn. J. Appl. Phys. 2019, 58, SBBJ02. [CrossRef]

16. Palani, I.A.; Vasa, N.J.; Singaperumal, M. Crystallization and ablation in annealing of amorphous-Si thin film on glass and crystalline-Si substrates irradiated by third harmonics of $\mathrm{Nd}^{3+}$ : YAG laser. Mat. Sci. Semicon. Proc. 2008, 11, 107-116. [CrossRef]

17. Nadarajah, A.; Könenkamp, R. Laser annealing of photoluminescent ZnO nanorods grown at low temperature. Nanotechnology 2010, 22, 025205. [CrossRef]

18. Meyer, F.; Büchler, A.; Brand, A.A.; Dasa, M.K.; Nekarda, J.F.; Preu, R. Impact of solidification dynamics on crystal properties of silicon molten by a nanosecond laser pulse. Appl. Phys. A 2018, 124, 254. [CrossRef]

19. Pyo, J.; Ryu, H.Y.; Park, J.; Lee, M.; Ryu, H.Y. Laser-power dependence of poly-silicon crystallization using 355-nm nanosecond laser annealing. J. Korean Phys. Soc. 2020, 76, 1116-1120. [CrossRef]

20. Nocuchi, T.; Chen, Y.; Miyahira, T.; Mugiraneza, J.D.; Ogino, Y.; Lida, Y.; Sahota, E.; Terao, M. Advanced micro-polycrystalline silicon films formed by blue-multi-laser-diode annealing. Jpn. J. Appl. Phys. 2010, 49, 03CA10. [CrossRef]

21. Jin, S.; Hong, S.; Mativenga, M.; Kim, B.; Shin, H.H.; Park, J.K.; Kim, T.W.; Jang, J. Low temperature polycrystalline silicon with single orientation on glass by blue laser annealing. Thin Solid Films 2016, 616, 838-841. [CrossRef]

22. Choi, Y.H.; Ryu, H.Y. Formation of a polycrystalline silicon thin film by using blue laser diode annealing. J. Korean Phys. Soc. 2018, 72, 939-942. [CrossRef]

23. COMSOL Multiphysics. Available online: https:/ /www.comsol.com/products (accessed on 18 September 2020).

24. Amin, M.S.; Hozhabri, N.; Magnusson, R. Effects of solid phase crystallization by rapid thermal annealing on the optical constants of sputtered amorphous silicon films. Thin Solid Films 2013, 545, 480-484. [CrossRef]

25. Moschou, D.C.; Vourdas, N.; Davazoglou, D.; Kouvatsos, D.N.; Vamvakas, V.E.; Voutsas, A.T. On the optical properties of SLS ELA polycrystalline silicon films. Microelectron. Eng. 2012, 90, 69-71. [CrossRef]

26. Kemiha, A.; Birouk, B.; Raskin, J.P. Ellipsometry-based conductivity extraction in case of phosphorus doped polysilicon. J. Mater. Sci. Mater. Electron. 2018, 29, 11627-11636. [CrossRef]

27. Hong, W.E.; Ro, J.S. Kinetics of solid phase crystallization of amorphous silicon analyzed by Raman spectroscopy. J. Appl. Phys. 2013, 114, 073511. [CrossRef]

28. Jin, J.; Yuan, Z.; Huang, L.; Chen, S.; Shi, W.; Cao, Z.; Lou, Q. Laser crystallization of amorphous silicon films investigated by Raman spectroscopy and atomic force microscopy. Appl. Surf. Sci. 2010, 256, 3453-3458. [CrossRef]

29. Wei, W.; Xu, G.; Wang, J.; Wang, T. Raman spectra of intrinsic and doped hydrogenated nanocrystalline silicon films. Vacuum 2007, 81, 656-662. [CrossRef]

30. Pyo, J.; Ryu, H.Y. Comparative study on crystallinity of laser-annealed polysilicon thin films for various laser sources. Mater. Express 2021, 11, 1239-1244. [CrossRef] 
31. Calcagnile, L. Laser induced phase transitions in Si and Ge implanted Si substrates. Phys. Stat. Solidi A 1995, 151, 23-32. [CrossRef]

32. Theodorakos, I.; Raptis, Y.S.; Vamvakas, V.; Tsoukalas, D.; Zergioti, I. Laser annealing and simulation of amorphous silicon thin films for solar cell applications. Proc. SPIE 2014, 8967, 89670T.

33. Caninenberg, M.; Verheyen, E.; Kiesler, D.; Stoib, B.; Brandt, M.S.; Benson, N.; Schmechel, R. Sample temperature profile during the excimer laser annealing of silicon nanoparticles. Opt. Laser Technol. 2015, 74, 132-137. [CrossRef]

34. Saprykin, A.A.; Ibragimov, E.A.; Babakova, E.V. Modeling the temperature fields of copper powder melting in the process of selective laser melting. IOP Conf. Ser. Mater. Sci. Eng. 2016, 142, 012061. [CrossRef]

35. Shrestha, M.; Wang, K.; Zheng, B.; Mokrzycki, L.; Fan, Q. Comparative Study of Furnace and Flash Lamp Annealed Silicon Thin Films Grown by Plasma Enhanced Chemical Vapor Deposition. Coatings 2018, 8, 97. [CrossRef]

36. Snyder, P.G.; Xiong, Y.-M.; Woollam, J.A.; Krosche, E.R.; Strausser, Y. Characterization of polycrystalline silicon thin-film multilayers by variable angle spectroscopic ellipsometry. Surf. Interface Anal. 1992, 18, 113-118. [CrossRef]

37. Valyukh, I.; Green, S.; Arwin, H.; Niklasson, G.A.; Wäckelgård, E.; Granqvist, C.G. Spectroscopic ellipsometry characterization of electrochromic tungsten oxide and nickel oxide thin films made by sputter deposition. Sol. Energy Mater. Sol. Cells 2010, 94, 724-732. [CrossRef]

38. Gong, J.; Dai, R.; Wang, Z.; Zhang, C.; Yuan, X.; Zhang, Z. Temperature dependent optical constants for SiO2 film on Si substrate by ellipsometry. Mater. Res. Express 2017, 4, 085005. [CrossRef] 